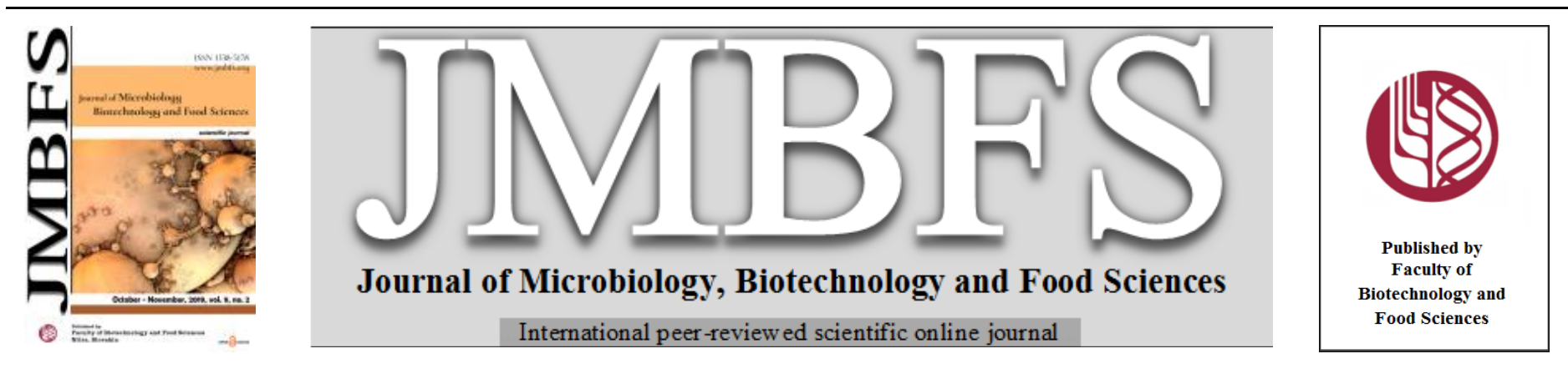

\title{
A MINI-REVIEW ON BIOFUELS FROM CHLAMYDOMONAS REINHARDTII
}

\section{Asmaa Missoum*}

Address(es):

Department of Biological and Environmental Sciences, College of Arts and Sciences, Qatar University, Doha City, Qatar.

*Corresponding author: amissoum93@gmail.com

doi: 10.15414/jmbfs.2019.9.2.273-279

ARTICLE INFO

Received 5. 7. 2018

Revised 24. 4. 2019

Accepted 26. 4. 2019

Published 1. 10. 2019

Regular article

open ${ }_{\text {ACCESS }}$

\begin{abstract}
This bibliographic review gives a concise overview of cultivation of microalgae for the production of biofuel. Chlamydomonas reinhardtii is one of the best microalgae that can be used for producing biofuel because of its simplicity of genome that allows it to be easily manipulated by genetic engineering for abundant growth. C. reinhardtii produces lipids under nitrogen deficient condition, which contains many triacylglycerols (TAGs) that influences the metabolism to a great extent and used for membrane remodeling. Several genes such as citrate synthase and DGAT2 involved in this mechanism were manipulated by engineering metabolic pathways to maximize lipid production under nitrogen deprived conditions. Additionally, C. reinhardtii can be efficiently cultivated and grown in closed photobioreactors, flat plate, tubular or vertical column with protected $\mathrm{CO}_{2}$ rather than open pond systems. In addition, $C$. reinhardtii can be harvested by several methods including Tris-Acetate Phosphate Pluronic (TAPP) medium method. Compared to conventional diesel, microalgae based biofuel, which is produced by trans-esterfication reactions, has a higher pour point temperature but similar properties. Contrasting Energy-efficiency ratio (EER) values of C. reinhardtii with those of other crops (Jatropha, palm oil and sunflower) is a crucial process before commercialization. The environmental impacts of microalgae are also beneficial in a way it can treat wastewater containing large amounts of nutrients such as nitrogen and phosphorus, which are beneficial for algae cultivation and produce bio-oil. However, this is a risky proposition as it can cause eutrophication and hypoxia in the ponds. Nevertheless, further research on bio-fuel production from $C$. reinhardtii must be carried out.
\end{abstract}

Keywords: Chlamydomonas reinhardtii, Biofuel, Microalgae, TAGs, TAPP, EER

\section{INTRODUCTION}

A "biofuel" is a type of fuel that is derived from living organisms such as plants, fungi, or algae. Research on these types of fuels has evolved since the past three decades as they offer an alternative source of sustainable energy compared to fossil fuels, which are depleting and causing huge environmental concerns such as the green house gases (Pandey $\boldsymbol{e t}$ al., 2014). Because extracting sources of energy such as biofuel from living organisms requires series of processes, biotechnology play an essential role in this field (Radakovits et al., 2010). This technology involves the utilization and manipulation of biological processes in microorganisms for industrial purposes such as the production of hormones, antibiotics, and biofuels. Few of the sources of biodiesel that caught many researchers' attention due to its various advantages are algal species (Lam and Lee, 2012).

Algae are photosynthetic microorganisms that either belongs to prokaryotes or eukaryotes. They range from unicellular structure including some filamentous and colonial algae (Microalgae) to simple multi cellular structure (Macroalgae). There are more than 50,000 of microalgae species living in various ecosystems including terrestrial and aquatic ones (Brennan, 2010). The cultivation of microalgae as source of biomass has many advantages such as they have higher capacity to capture sunlight and use it for photosynthesis in order to produce organic compounds (Mata et al., 2010). Moreover, microalgae produce higher amounts of polymers, pigments, carbohydrates, lipids, and proteins when they are subjected to chemical and physical stress conditions. Compared to other microorganisms, many microalgae possess simple cellular division cycle without a sexual stage, which allows them to grow rapidly and eventually produce high biomass yield. Finally, because microalgae live in all kinds of environments differing in temperature, salinity, and light intensities, it is easier to harvest ones with prefered properties such as tolerating extreme conditions (Pandey $\boldsymbol{e t}$ al., 2014). Thus, they do not need mass areas of lands or fresh waters as they can even grow in sewage or waste water. Other facts that must be taken into consideration are: microalgae-based fuels do not compete with food supply such as corn and soybeans based biofuels, are renewable, and can be carbon reducing.
In fact, generating 100 tons of algae biomass is equivalent to removing about 183 tons of carbon dioxide from the atmosphere (Pandey et al., 2014).

Chlamydomonas reinhardtii is considered one of the best candidates for biofuel production. Some of the various reasons are that this model organism has been the lead in development of molecular tools for engineering for most green alga thanks to its simple genome. In fact, $C$. reinhardtii was the first engineered algal species that was studied for commercial purposes, and it was stated that more recombinant proteins have been expressed in $C$. reinhardtii than the rest of all examined algal species (Radakovits et al., 2010). Moreover, studies on these microalgae have also managed to expose the molecular mechanisms behind algal lipid and hydrogen metabolism. This unicellular alga accumulates large amount of energy rich molecules such as triacylglycerols (TAGs) under unfavorable conditions (Nitrogen starvation). These are then converted to biodiesel by transesterification reactions (Lenka et al., 2016). However, there are several factors that make microalgae based biodiesel production economically and commercially unfeasible. Low light penetrating dense culture, lack of effective extraction methods, and difficulties in harvesting biomass at large scale all lead to lower oil content (Bellou et al., 2014). Therefore, it is crucial to use metabolic and genetic engineering to optimize this microalga's biomass and oil content production to be used for biofuels such as biodiesel (Deng et al., 2012; Deng $\boldsymbol{e t}$ al., 2013).

Many believe that ancient algae were responsible for creating crude oil deposits. Thus, it is clear that using best studied microalgae strains to synthesize biofuels should be a priority among other alternatives (Pandey et al., 2014). In this bibliographic review, we will be discussing some of metabolic applications to enhance Chlamydomonas reinhardtii lipid production as well as basic methods for cultivating and harvesting its biomass. We will also point out the basic principles of extracting oil from cells and trans- esterification reactions, as well as essential properties that makes microalgae oil appropriate for biofuel applications. 


\section{BIOFUELS FROM CHLAMYDOMONAS REINHARDTII}

\section{Metabolic Engineering and Applications}

Recent research in transcriptomics and metabolomics has managed to give better insights regarding molecular mechanisms involved in TAG synthesis. First, free fatty acids are produced in the chloroplast after converting pyruvate to Actyl CoA by pyruvate dehydrogenase (PDH). This is further converted to Malonyl CoA by acetyl-CoA carboxylase (ACCase), which is used by the enzyme malonyl-CoA:ACP transacylase (MAT) to synthesize Malonyl ACP (Bellou et al., 2014). This product is consequently involved in the fatty acid synthesis cycle. Accordingly, after releasing fatty acids into the cytosol, they are converted into acyl CoA by acyl CoA synthetase, which is then involved in series of reactions by several enzymes on the endoplasmic reticulum (ER). This leads to assembly of triacylglycerols (TAGs) as shown in figure 1 (Bellou et al., 2014).

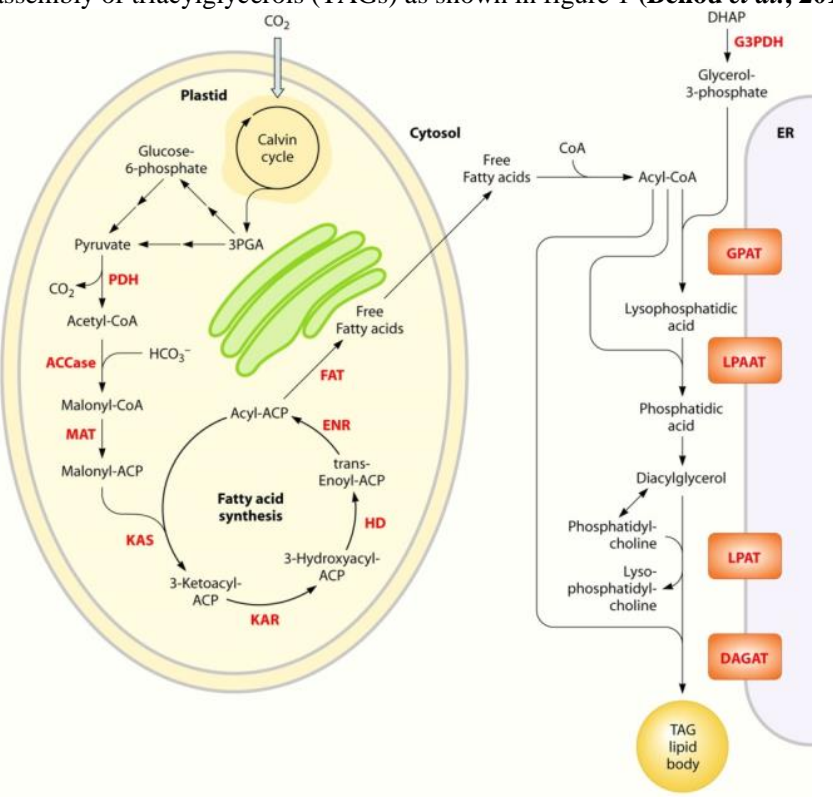

Figure 1 Summarizes lipid biosynthesis processes in Chlamydomonas reinhardtii. GPAT, glycerol-3-phosphate acyltransferase; LPAAT, lysophosphatidic acid acyltransferase; LPAT, lyso-phosphatidylcholine acyltransferase; DAGAT, diacylglycerol acyltransferase (Bellou et al., 2014).

After selecting strains with maximal TAGs production, out of few options investigated recently to enhance production of TAGs is to knockdown of the mRNA expression of the phosphoenolpyruvate carboxylase isoform 1 (CrPEPC1) gene by RNA interference. In this study, TAGs levels have increased by $20 \%$ (Deng et al., 2014). During Nitrogen deprivation conditions, genes involved in triacylglycerol (TAG) biosynthesis are up-regulated whereas those involved in photosynthesis, protein biosynthesis, and tricarboxylic (TCA) cycle are downregulated. Phosphoenolpyruvate carboxylase (PEPC) is an enzyme that catalyzes the formation of oxaloacetate (OAA) from phosphoenolpyruvate (PEP). This then enters the TCA cycle to provide the substrate for protein synthesis (Deng $\boldsymbol{e t}$ al. 2014). As mentioned earlier, acetyl-CoA carboxylase (ACCase) catalyzes the formation of malonyl-CoA from acetyl coenzyme A and consequently enters the fatty acid synthesis pathway. Therefore, the carbon flux from glycolysis synthesizes fatty acids or proteins depending on the activities of PEPC and ACCase as illustrated in figure 2A (Deng $\boldsymbol{e t}$ al., 2013).
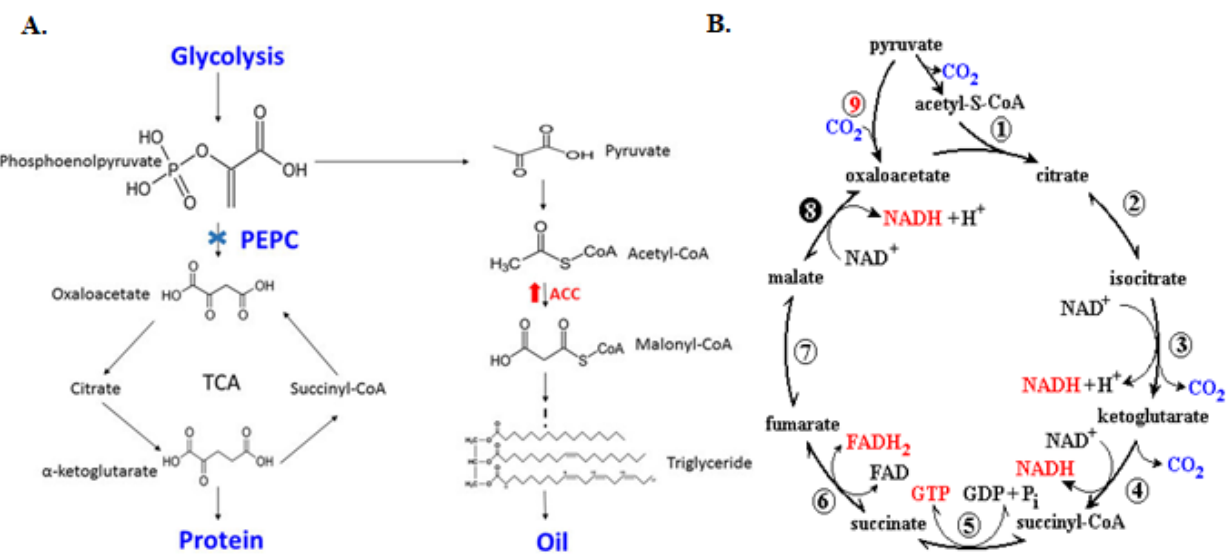

Figure 2 A Summarizes the distribution of carbon flow between protein and lipid synthesis pathways (Deng et al., 2014). B Summarizes the TCA cycle in which "1" refers to citrate synthase (Deng et al., 2013).

Given that both lipid and protein biosynthesis pathways share the same substrate; the deceased consumption of phosphoenolpyruvate of protein pathway helped to increase the final product (TAGs) in the lipid pathway. Thus, the downregualtion of PEPC using RNAi has lead to a decrease in carbon flow into the TCA cycle (Deng et al., 2014).

Similarly, another way of maximizing TAGs production is by the knockdown of citrate synthase gene. This gene, also known as "CrCIS", codes for the enzyme citrate synthase. Located in the mitochondria, the main function of citrate synthase in the TCA cycle is to catalyze acetyl CoA reaction to Citroyl CoA (See figure 2B). In this study, it was reported that TAGs levels were increased by $169.5 \%$ after silencing CrCIS using RNA interference. Thus, when the respective enzyme is not synthesized, acetyl CoA will accumulate as the TCA cycle is blocked and will be used instead in the lipid biosynthesis pathway. Moreover, silencing of this gene did not slow down cell proliferation (Deng et al., 2013).
TAGs levels can be also increased by over expressing genes CrDGAT2-1 and CrDGAT2-5. These are two of the five homologous genes responsible in coding for DGAT (diacylglycerol acyltransferase) enzymes of different families. These enzymes are involved in assembling TAGs (Final step in the lipid biosynthesis pathway) by covalently linking diacylglycerols (DAGs) to long-chain fatty acylCoAs. The over expression of CrDGAT2-1 and CrDGAT2-5 genes has lead to 49.75 and $59.01 \%$ increase in lipid content respectively (Deng $\boldsymbol{e t}$ al., 2012).

Moreover, it important to keep in mind that in previously mentioned studies, Chlamydomonas reinhardtii accumulated more TAGs content under nitrogen deprivation conditions. These conditions influence the metabolism to a great extent as acetate is directly funneled into fatty acid biosynthesis and further fatty acids are synthesized by membrane remodeling (Deng et al., 2013). Moreover, the breakdown of intracellular stores is a useful source of carbon for nitrogen reassimilation. Glycolysis genes are also up regulated regardless the decrease in photosynthetic carbon fixation. This indicates that parts of the carbon from starch are utilized for fatty acid biosynthesis (Deng et al., 2013). However, nitrogen deprivation also causes thylakoids/chloroplast breakdown, and thus 
almost completely prevents cell growth. Another current study showed that TAGs also accumulated under after phosphorus limitation conditions without affecting thylakoid membranes (Lenka et al., 2016). Latest research confirms that supplementing growth culture with $4 \mathrm{~g} / \mathrm{L}$ sodium acetate counters these effects the result in biomass reduction and increases fatty acids yield by $93.0 \%$ when combined with both nitrogen and phosphorous deficiency (Yang et al., 2018a).

Recently, new strategies were employed to maximise lipid productivity. Using ptxD gene expression from Pseudomonas stutzeri strain WM88, C. reinhardtii was capable of using phosphite as its only phosphorus source. This gene encodes for the enzyme phosphite oxidoreductase, which oxidizes phosphite into phosphate by NAD as a cofactor. This transgenic $C$. reinhardtii also outcompeted other microalgae and natural contaminants present in the system, meaning that there is no need to use antibiotics and herbicides for sterile conditions. This strategy was proven useful for both open pond systems and closed photobioreactors (Loera-Quezada et al., 2016)

Another way of optimizing lipid biosynthesis in $C$. reinhardtii is through induced regulation of two kinases: c-Jun N-terminal kinase (JNK) and extracellular signal regulated kinase (ERK). Under osmotic stress promoted by

$\mathrm{NaCl}$, ERK activation by $\mathrm{C} 6$ ceramide and lipid production has augmented by 1.44 and 1.7 fold, respectively. In contrast, inhibition of JNK pathway using SP600125 has resulted in $40.53 \%$ decrease cell growth at the same cultural conditions (Yang et $\boldsymbol{a l}$., 2018b). The mechanisms of these two kinases under osmotic stress and the effect of biochemical regulations from all experiments in the study are summerised in figure 3 .

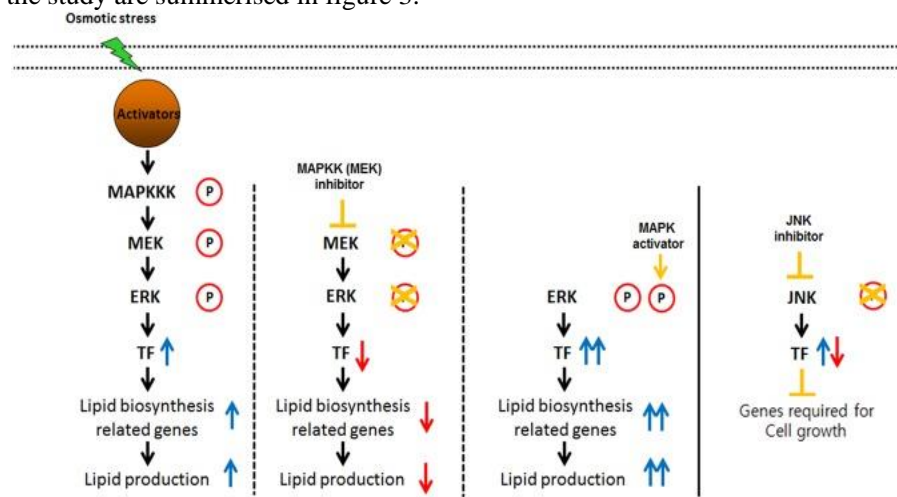

Figure 3 Summary of biochemical pathways involved in lipid biosynthesis and cell growth (yang et al., 2018b).

The microalga $C$. reinhardtii contains 17 bZIP (basic leucine-region zipper) transcription factors that are crucial in regulating stress responses which were identified in a novel study. Also known as CrebZIP, they could play a significan role in lipid accumulation. This was demonstrated under salt stress conditions promoted by $150 \mathrm{mM} \mathrm{NaCl}$ treatment, which increased lipid content from 0.284 \pm 0.029 to $0.437 \pm 0.012 \mathrm{~g} / \mathrm{g}$ after 48 hours (Ji et al., 2018). Using quantitative real time PCR analysis, it was found that only six CrebZIP were expressed such that the genes CrebZIP4,5, and 13 were down-regulated, whereas genes CrebZIP10, 11, and 16 were up-regulated. Therefore, it is possible to exploit the regulatory mechanisms of these CrebZIP genes in the future studies to engineer more efficient $C$. reinhardtii strains, which will eventually enhance biomass and biofuel production (Ji et al., 2018).

\section{Cultivation and Biomass Harvesting}

Chlamydomonas reinhardtii is grown in either open pond systems or in large vessels called photo-bioreactors. These must have $\mathrm{CO}_{2}$ and external light supplies, as well as large surface areas to guarantee enough light diffusion and $\mathrm{CO}_{2}$ in the media. In open pond systems, ponds are constructed in shallow dimension to allow the microalgae to use more light as possible (Park $\boldsymbol{e t}$ al. 2011). However, these require large areas of lands and because they are open, $\mathrm{CO}_{2}$ is lost to the atmosphere. Moreover, uncontrolled growth environmental
A.

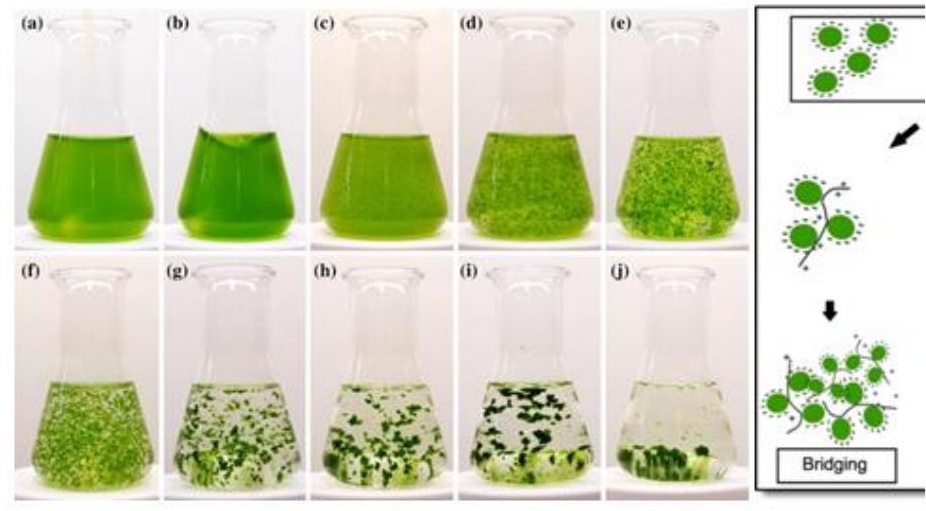

Figure 4 A Shows stages of coagulation of microalgal cells (Salim et al., 2010). B Summarizes two mechanisms of flocculation: bridging and patching (Bleeke et al., 2015).

factors such as temperature might lead to contamination, poor light diffusion, and evaporation (Park et al., 2011).

In closed photo-bioreactors, all conditions are controlled. This gives advantages as undisclosed $\mathrm{CO}_{2}$ is not lost to the atmosphere and the partial pressure is easily maintained. One commonly used type of these systems is the vertical tubular photo-bioreactor. These are made of transparent tubes that allow more light penetration. At the bottom of each tube, a sparger is attached to convert sparged gas into tiny bubbles and removed produced $\mathrm{O}_{2}$ during photosynthesis. Mixing in these systems is achieved mechanically by a paddlewheel and using gas flows respectively. This helps to distribute metabolite, cells, and gases homogenously in the media. It is also important to consider that the optimum conditions for cultivation varies for one strain to another and depends on which system is used. Generally, keeping $\mathrm{pH}$ at 7.5 and using moderate $\mathrm{CO}_{2}$ levels will enhance cultivation (Pandey et al., 2014).

The first step of the microalgae harvesting is screening. Here, the biomass is introduced into a screen in order to filter anything else and capture only microalgae cells. This can be accomplished by various methods including the use of micro-strainers or vibrating screens. The following step requires the use of chemicals that induce coagulation and flocculation (Salim et al., 2010). This causes microalgae cells to aggregate into clamps that can be filtered easily from the medium (Figure 4A). In order to achieve coagulation, two types of chemicals are used: inorganic coagulants such as metal ions $\mathrm{Fe} 3^{+}$and $\mathrm{Al}^{+}$from Aluminum and Ferric sulphates, and long chain organic coagulants like poly-electrolytes and natural polymeric substances. The former type are cations that bind to negatively charged microalgae particles and thus neutralize the environment such that they do not repel each other anymore and therefore clump together. Similarly, flocculation using polymers causes cells to bind to positively charged polymers. If they bind partly, the unoccupied spaces of polymers can bind other cells and consequently bridge them. However, if they bind completely, they patch to the surface creating local positive charges that can attract other microalgae cells resulting in flocculation (Bleeke $\boldsymbol{e t}$ al., 2015). This is illustrated in figure $4 \mathrm{~B}$.

In order to harvest the small sized microalgae such as $C$. reinhardtii, a method called floatation is used. Its main principle is based on that fact that when solid particles attach to air bubbles, they result in flocs floating the broth or liquid surface because of buoyancy (Chen et al., 2011). This method costs a lot as it requires bubbling air through a column, using maximum amount of coagulants, and adjusting other factors such as $\mathrm{pH}$. Bulk harvesting methods (flocculation) uses low energy that can be cost effective to harvest $C$. reinhardtii. Iron chloride $\left(\mathrm{FeCl}_{3}\right)$, aluminium sulphate $\left(\mathrm{Al}_{2}\left(\mathrm{SO}_{4}\right)_{3}\right)$, and iron sulphate $\left(\mathrm{Fe}_{2}\left(\mathrm{SO}_{4}\right)_{3}\right)$ are conventional flocculants (Brennan and Owende, 2010) that transfer microalgal cells to slurry to stay out of the cultivation medium. Filtration can then release water from the slurry to concentrate the amount of microalgal slurry. Development of biodegradable and harmless organic flocculants can be used for harvesting algae (Suali and Sarbatly, 2012)

Gravity sedimentation is another method to separate microalgal cells such that this time they settle to the bottom out of the fluid responding to forces of gravity. However, because the microalgae have small size, the settling rate is too low and slow (Bux, 2013). For this reason, this rate is increased by centrifugational force. Centrifugation is very effective as it was reported that it results in 90 $100 \%$ harvesting efficiency but it is also cost and energy intensive (Pandey $\boldsymbol{e}$ al., 2014). 
Although research on improving previously mentioned harvesting methods continues, other new strategies and devices are invented. Recently, researchers cultured Chlamydomonas reinhardtii in a Tris-Acetate Phosphate Pluronic medium, also known as TAPP successfully without affecting the productivity (Estimeet et al., 2017). This medium undergoes a thermoreversible sol-gel transition, meaning that after culturing microalgae in the TAPP medium in a solution phase at $15{ }^{\circ} \mathrm{C}$, the temperature is increased at $22{ }^{\circ} \mathrm{C}$ to cause medium gelation and trap microalgal cells as illustrated in figure 5 .

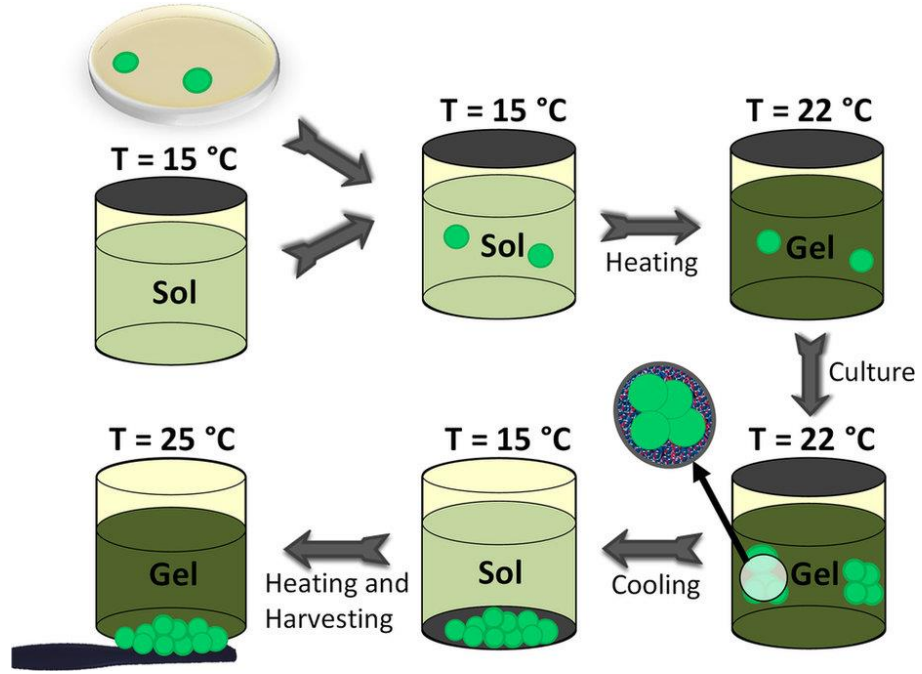

Figure 5 Summary of cultivation and harvesting Chlamydomonas reinhardti cells stages using thermo-reversible gel and Tris-Acetate Phosphate Pluronic (TAPP) medium (Estimeet et al., 2017).

After the cultivation period, the temperature is lowered to $15^{\circ} \mathrm{C}$ allowing microalgal clusters to gravimetrically settle at the bottom. Finally, the temperature was increased to jellify the supernatant and harvesting of the microalgae simply involved scraping microalgal flocs off the TAPP surface. The whole process is repeatable and takes only 2 hours without further requirement of additional materials. Another advantage of the TAPP medium is that it can be reused and recycled medium for re-cultivation of microalgae after the harvesting process (Estime et al., 2017).

In order to improve biomass production, old concepts of crops enhancement can be used instead of DNA genetic techniques that are used to boost lipid production. In a novel study, diploidy was induced in $C$. reinhardtii by treating the haploid cells with colcemid, a microtubule inhibitor. Then, two diploid strains, CMD ex1 and CMD ex4, were selected. These coped well when exposed to cold, nutritional, and oxidative stresses, as well as accumulated biomass and Fatty acids yield by two fold when compared to the control (Kwak et al., 2017). Another strategy involves the use of microalgae growth promoting bacteria (MGPB) as 'probiotics'. These were obtained from wastewater effluent samples in Yamanashi, Japan and were tested on three different microalgae including $C$. reinhardtii. The bacteria enhanced their host's biomass production by nearly 1.5 fold after 7 days. Using quantitative PCR and 16S rRNA gene analysis, the classes of bacteria were identified as Alphaproteobacteria, Betaproteobacteria Sphingobacteriia and Saprospirae (Toyama et al., 2018)

\section{Preparation of Chlamydomonas reinhardtii Oil as Biofuel}

This is an important multi step process that depends heavily on how biomass was previously harvested. For this reason, selecting best method is very crucial as it consequently affects biofuel production. First, the biomass is dried to avoid moisture interference with solvents. Cells are then disrupted by various methods such as squeezing cells under high pressure (Expeller pressing) or using beads (Bead beating) in order to get intracellular contents to the solvent medium. Afterwards, algal oil is extracted by many methods (Bellou et al., 2014). One of these methods is solvent extraction as lipids are soluble in organic solvents. Lipids' solubility plays a major role as each value depends on the type of solvent that is more suitable to it. For example, polar lipids such as Phospholipids dissolve in polar solvents and non-polar lipids such as triglycerides dissolve nonpolar solvents (Lam and Lee, 2012). Enzymatic treatment could be also used to break down cell wall with water acting as solvent and thus oil content separates during the process. However, this is not feasible for large scale application. Other methods including osmotic shock, pulse electric field technology, and hydrothermal liquefaction are highly expensive and necessitate further optimization efforts (Suali and Sarbatly, 2012).

Transesterification is a reversible process where one mole of triacylglycerides is reacted with three moles of alcohol, usually methanol or ethanol, as well as adding a catalyst (See figure 6) to synthesize glycerol as byproduct and fatty acid methyl esters (FAME). The latter are then used as biodiesel. In an acid catalyzed transesterification, the reaction is carried at $80^{\circ} \mathrm{C}$ at a slow rate requiring high amounts of alcohol. One example of the catalyst used is $\mathrm{H}_{2} \mathrm{SO}_{4} / \mathrm{HCl}$. However, in an alkali catalyzed one, it is completed at $60^{\circ} \mathrm{C}$ in 90 minutes requiring the use of potassium and sodium hydroxides as catalysts (Pandey et al., 2014). This process is 4000 times faster but water removal is a crucial step before beginning as it causes base hydrolysis. Another type of transesterification reaction is one that is catalyzed by lipases enzymes as they convert all triglycerides into biodiesel. This process is extremely unfeasible as it requires complex processing (Brennan and Owende, 2010).

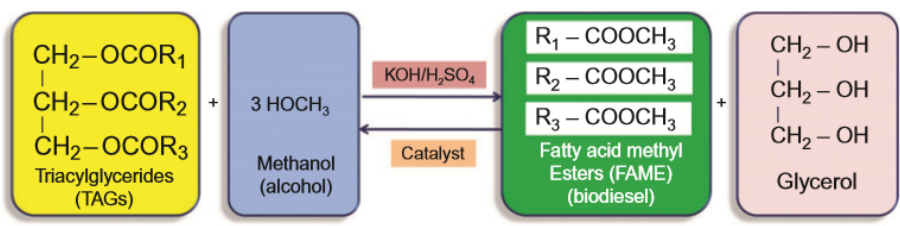

Figure 6 Summary of acid or base catalyzed trans-esterfication process of triacylglycerides (TAGs) with methanol giving glycerol and fatty acid methyl esters (FAME) (Pandey et al., 2014).

In order for fatty acid methyl esters to be used as biodiesel, certain characteristics such as iodine and acid number, flash and pour points, and kinematic viscosity must be examined. The iodine number refers to the unsaturation degree and acid number indicates the corrosives of oil. The flash point is the lowest temperature at which oil vaporizes to potentially form ignitable mixtures and that of biodiesel is much higher than conventional diesel (Pandey et al., 2014). Additionally, pour point is the lowest temperature at which oil loses its flow properties and becomes semi-solid. This is a vital property to qualify microalgae oil as a diesel because its pour point is slightly higher than that of conventional diesel. This is useful in countries with tropical hot weather such as India. Other important characteristics include heating value and cetane number. The former is defined as the energy released as heat when the compound undergoes combustion and the latter refers to ignition quality (Suali and Sarbatly, 2012). All properties of both microalgae biodiesel and conventional diesel are compared in summarized in table 1 below, suggesting that it is a good candidate for biofuel applications (Brennan and Owende, 2010).

Table 1 Comparison between microalgae biodiesel and conventional diesel fuel properties (Pandey, 2014).

\begin{tabular}{lllll}
\hline S. No. & Fuel Property & $\begin{array}{l}\text { Biodiesel Standarc } \\
\text { (ASTM) }\end{array}$ & $\begin{array}{l}\text { Microalga } \\
\text { Biodiesel }\end{array}$ & $\begin{array}{l}\text { Diesel Fue } \\
\text { (ASTM) }\end{array}$ \\
\hline $\mathbf{1}$ & $\begin{array}{l}\text { Acid number } \\
(\mathrm{mg} \mathrm{KOH} / \mathrm{g} \text { of oil) }\end{array}$ & $<0.5$ & 0.42 & $0.7-1.0$ \\
$\mathbf{2}$ & $\begin{array}{l}\text { Iodine value } \\
(\mathrm{g} \mathrm{I} / 100 \mathrm{~g} \text { of oil) }\end{array}$ & $<25$ (efficient fuel) & 19.0 & 120 \\
& Specific gravity $\left(\mathrm{g} / \mathrm{cm}^{3}\right)$ & $0.85-0.90$ & 0.85 & $0.82-0.90$ \\
$\mathbf{3}$ & Density $\left(\mathrm{g} / \mathrm{cm}^{3}\right)$ & 0.88 & 0.85 & $0.86-0.90$ \\
$\mathbf{4}$ & $\begin{array}{l}\text { Kinematic viscosity } \\
\left(\text { mm }^{2} / \mathrm{s}\right)\end{array}$ & $1.9-6.0$ & $2.0-4.5$ & 3.5 \\
$\mathbf{5}$ & Heating value $(\mathrm{MJ} / \mathrm{kg})$ & 44 & 37 & 42.2 \\
$\mathbf{6}$ & Flash point $\left({ }^{\circ} \mathrm{C}\right)$ & 130 & $>130$ & $>62$ \\
$\mathbf{7}$ & Pour point $\left({ }^{\circ} \mathrm{C}\right)$ & -11.6 & -6 & -16 \\
$\mathbf{8}$ & Cetane number & 47 & 46 & 60 \\
\hline $\mathbf{9}$ & & & & \\
\hline
\end{tabular}

\section{Scale up and Commercialization}

As mentioned earlier, cultivating $C$. reinhardtii for biodiesel production has many advantages. These include the fact that it is fast growing and have 100 times higher reproducibility than land plants. In addition, it accumulates lipids as $75 \%$ of its total weight, and unlike plants, agricultural lands are not needed to cultivate it. Large-scale cultivation of $C$. reinhardtii faces numerous technical challenges that slow down the commercialization of renewable microalgae feedstock for producing biodiesel (Lam and Lee, 2014). These consist of upstream processes such as selecting $C$. reinhardtii strains for cultivation, nutrient sources, minimum energy input for closed photo-bioreactors, reusing water and sensitivity of microalgae for surrounding the environment. Other challenges include downstream processes such as harvesting and drying $C$. reinhardtii cells, conversion of microalgal biodiesel with good quality, and effective techniques for lipid extraction. Finding solutions for these challenges 
could enhance the economic feasibility of microalgae-based biodiesel production (Lam and Lee, 2014).

A minimum input of energy and cost-effective protocols are the first challenges to consider when commercializing algal biofuels. Cultivation systems need to have large areas of light, utilize optimal gas-liquid transfer, low or no contamination and must be easy to operate (Xu et al., 2009). The preferred technique of cultivating $C$. reinhardtii is phototropic cultivation, and it requires a high intensity of sunlight and it recaptures $\mathrm{CO}_{2}$ from flue gases. This method has limitations if sunlight is not very radiant in case of temperate countries (Lam and Lee, 2012). Moreover, phototropic $C$. reinhardtii can grow in open pond system and closed photo-bioreactor. Considering the issue of energy balance for producing microalgal biofuels, when it is cultivated in closed photo-bioreactors, studies by life-cycle assessments (LCAs) of $C$. reinhardtii biodiesel production showed that there was a negative energy balance in cultivation (Jorquera et al. 2010; Razon and Tan, 2011; Stephenson et al., 2010).

Energy-efficiency ratio (EER) can improve the energy conversion efficiency of algal biofuel production before commercialization. The EER is the ratio that represents total output of energy to total input of energy. If the ratio is higher than 1 , then net positive energy is produced. Table 2 shows a study on EER for biodiesel originated from Jatropha, palm oil, sunflower, and Chlamydomonas reinhardtii. The values are an estimated indication based on different assumptions from studies of the LCA. In Table 2 , microalgae $(C$. reinhardtii) contains the highest EER out of the feedstock crops used, which means the energy required for microalgae cultivation requires high energy input to produce biofuel. Moreover, the cultivation of $C$. reinhardtii for producing biodiesel may create a progressive energy output (Lam and Lee, 2012).

Table 2 Energy-efficiency Ratio (EER) for Algae and crops (Lam and Lee, 2012)

\begin{tabular}{|c|c|c|}
\hline Feedstock & EER & Comment \\
\hline Jatropha & 3.40 & Included biogas production \\
\hline Palm Oil & 3.53 & co-product \\
\hline Sunflower & 3.50 & Based on Chilean conditions \\
\hline $\begin{array}{l}\text { Chlamydomonas } \\
\text { reinhardtii }\end{array}$ & 4.34 & $\begin{array}{l}\text { Low nitrogen culture and } \\
\text { biomass are not droed for } \\
\text { extraction }\end{array}$ \\
\hline
\end{tabular}

Closed photo-bioreactors are widely used for $C$. reinhardtii cultivation. They are designed to ensure that the microalgae will be produced at optimal conditions with high stability with a minimum level of contamination. Water sources are reutilized for cultivation cycles. Physiological and biological factors such as $\mathrm{pH}$, concentration of $\mathrm{CO}_{2}$, and nutrient level can optimize cultivation of $C$. reinhardtii strains in closed photo-bioreactors. A closed photo-bioreactor always produces high biomass productivity of $0.05-3.8 \mathrm{~g} / \mathrm{L} /$ day with the mass ratio of $0.00035-0.027$ of $C$. reinhardtii to water in 7 days when conditions of the cultivation and algal strains are controlled and optimized (Brennan and Owende, 2010; Lam and Lee, 2012).

Examples of closed photo-bioreactors are flat plate, tubular and vertical column Figure 6 outlines the structures of the three examples. Flat plate is a rectangular structure that has a large area of radiating sunlight and utilizing solar energy and uses a gas pipe that contains low dissolved oxygen, although it is difficult in controlling temperature of culture (Lam and Lee, 2012). Tubular method consists of looped transparent tubes that has a large area same as flat plates that uses sunlight for energy and processing, they produce a high biomass of the microalgae and reduce cell damage, although mixing can be challenging when products appear in whole tube, also long tubes are used so cultivation requires a vast area. The vertical column is a vertically rearranged cylinder column that have a high mass area that mixes $C$. reinhardtii very well for cultivation, its columns are made of transparent glass and the method is compact and easy to operate, also it is inexpensive (Lam and Lee, 2012).

Table 3 Examples of closed photobioreactors and their structures (Chen et al., 2011).

\begin{tabular}{|l|c|}
\hline Closed Photo-Bioreactor & Structure \\
\hline Flat plate & \\
\hline Tubular & \\
\hline & \\
\hline
\end{tabular}

There are upstream and downstream processes of $C$. reinhardtii cultivation. One of the upstream processes of algal cultivation includes water reusability and footprint (WF). Water footprint is when an area contains the total amount of fresh water to form a product for consumers (Mata et al., 2010). Production of biofuel requires $1 \mathrm{~L}$ of microalgal biodiesel and $3000 \mathrm{~L}$ of water, and water is recycled for cultivation of microalgae to lessen the water demand. Water demand is the difference between the required water for cultivation and the annual precipitation (Xu et al., 2009). If lipid productivity is low in a $C$. reinhardtii strain for biofuel production then area of cultivation is highly required. Lipid productivity in microalgae to produce biofuel was discussed earlier. $C$. reinhardtii are reproduced in water when in culture medium; noncellular water of $200 \mathrm{~kg}$ is needed for growing microalgae for cultivation (Xu et al., 2009).

One of the downstream processes of $C$. reinhardtii cultivation is harvesting the microalgal biomass, which is separating algae from water to produce biofuel. The first step is bulk harvesting, to separate the microalgae from suspended bulk in gravity sedimentation, flocculation, and flotation and the second step is to thicken and concentrate the microalgae after harvesting bulk by filtration and centrifugation as discussed earlier (Mata $\boldsymbol{e t}$ al., 2010). It is difficult to harvest the microalgal cells and suspend them in water, because they have a small size of $1-$ $20 \mathrm{~mm}$. Water of 73 tonnes need to be treated when harvesting the microalgal biomass of 1 tonne for cultivation. This develops effective $C$. reinhardti harvesting for strengthening the commercialization of microalgal biofuel production (Mata et al., 2010).

\section{Economics}

There are three major contributions to production cost: depreciation, raw materials and utilities, and labor and supervision. Figure 7 shows a flowchart of the capacity of the production and parameters of the distinctive developed operations. It provides the available type and size as well as energy and mass of equipment (Acien et al., 2014).

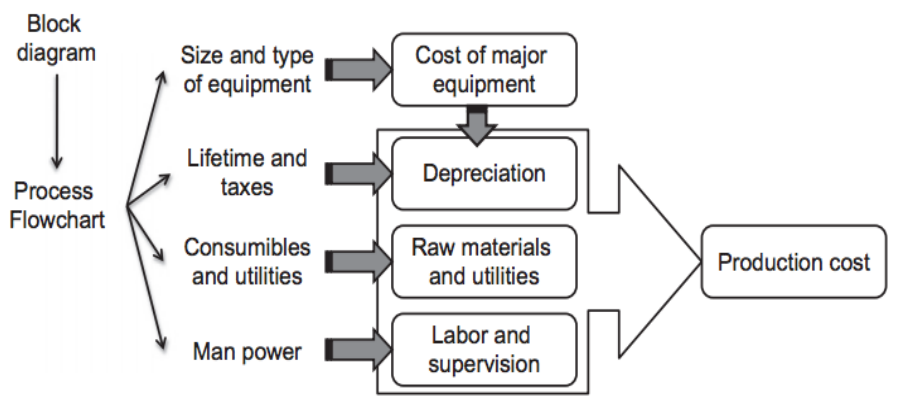

Figure 7 Flowchart of the cost analysis for $C$. reinhardtii production (Acien et al., 2014).

According to the flow chart, the required raw materials are calculated from mass balances. The utilities and consumption is calculated from use of power and water. The cost of raw materials includes the suppliers' market values and facility transport. With regard to power, the cost of electricity can vary according to consumption and energy required; therefore, a detailed analysis of different suppliers is recommended (Acien et al., 2014).

Cities with high pollution can generate high amounts of wastewater and it provides as a fertilizer. Untreated or treated wastewater can replace fertilizers and are cost effective; they also form a revenue stream to compensate the amount of bio-crude. Reducing the nutrient level satisfactorily minimized pollution of wastewater in China lakes and rivers, and for a while the lake will purify itself. Consequently, treating wastewater for treatment plant can produce growing diatoms. Treatment plant converts organic nutrients to inorganic ones Economically viable products can be made from integrated system of growing diatoms in producing fuel. Fertilizers are the most expensive cost of $C$. reinhardtii based biofuels (Acien et al., 2014; Mata et al., 2010].

Moreover, 5kt per year of $C$. reinhardtii is produced internationally in markets. The price ranges from $€ 10-300 / \mathrm{kg}$ and the market size is $10-50 \mathrm{kt} /$ year. Moreover, with high salary levels cost of labor of workers that are managing and supervising the process increases. For establishing cost production of biomass of $C$. reinhardtii used for biofuel, modifying the cost of the scaled equipment is used and multiplying the direct cost by the suitable factor of 10 raises the capacity, in which the formula can be simplified as $\operatorname{CostB}=\operatorname{Cost} A$ (SizeB / SizeA) scale-up factor (Acien et al., 2014)

\section{Environmental Impacts}


Environmental impacts of $C$. reinhardtii biofuels have its benefits and drawbacks. Depending on the input resources and the cultivation, $C$. reinhardtii biofuels could either damage quality of water or enhance it. The microalgae remove nutrients from waste by their potential water-quality that reduces eutrophication and runoff of herbicides and insecticides. Therfore, it is more beneficial in their water quality than corn-based ethanol and soybean-based biodiesel. The effects of water quality depend on the amount of nutrient of the microalgal culture medium, whether systems of feedstock are covered from heavy rain. Floods or culture fluid leakage to groundwater can happen if the reliability of the pond is compromised although these downfalls can be prevented with good managing of the culture processes (Bellou et al., 2014).

In $C$. reinhardtii cultivation, water can be re-used for easier cultivation. The liquid waste can be re-used to form biogas from the anaerobic respiration (Davis et al., 2011). In addition, released waters from $C$. reinhardtii cultivation aquifers are saline. The Clean Water Act and National Pollutant Discharge Elimination System can operate facilities of the microalgae cultivation. Releasing harvest water for terrestrial crops can be against the law for using it for biofuel factories. Moreover, leaching to groundwater could cause matters of $C$. reinhardtii cultures to be lost this can be caused by wind and high precipitation. Unintentional spills of cultivation water can be caused by environmental hazards such as hurricane, tornadoes or even earthquakes. Producers can deal with unintentional spills of cultivation water by defensive measures for predicting when hazardous weather events would take place, so that it does not affect their income (Bellou $\boldsymbol{e t}$ al., 2014).

Large amounts of nitrogen and phosphorus are needed for a high yield of large scale cultivation of $C$. reinhardtii. Accidental spills of cultivation water causes eutrophication of freshwaters and marine waters. Eutrophication signifies microalgal growth caused by presence of high concentrations of nitrogen and phosphorus in ponds. When $C$. reinhardtii decompose in the ponds generating high organic matter will lower the oxygen level in the water, which can lead to hypoxia in the ponds (Rabalais et al., 2009; Smith and Schindler, 2009). In some cases, eutrophication-induced changes could be difficult or impossible to reverse if alternative stable states can occur in the affected ecosystem (Carpenter, 2005).

C. reinhardtii have been also involved in treating wastewater, where they provide photosynthetically produced oxygen for the bacteria, which breakdown organic compounds present in the waste. High rate algal ponds (HRAPs) treat agricultura wastewater and growth of photosynthetic microalgae (Park et al., 2011). HRAPs remove nitrogen and phosphorus from the wastewater by algae. Additionally, in wastewater treatment, phosphorus is precipitated by insoluble solid (aluminum or iron-based coagulants) or it is unprotected from the water by microbial activity. The phosphorus when developed forms a sludge fertilizer. It is a beneficial process in lab experiments to recycle nutrients from wastewater and use them for further C. reinhardtii production (Sturm and Lamer, 2011)

\section{CONCLUSION}

Chlamydomonas reinhardtii is essential by many aspects for producing biofuels. C. reinhardtii is easily used for genetic engineering from its simple genome and produces lipids for biofuel. The partial pressures are constant for sustainability. Also C. reinhardtii compared to other conventional diesel have better properties such as a high point temperature for greater biofuel production. Additionally, the microalga has a higher energy-efficiency ratio (EER) than other crops used for biofuels for better cultivation and efficiently producing biofuel. Recommendations of producing biofuels from microalgae are using biodiesel to exchange fossil-based petroleum, because it is easier for lipid extraction to produce it, using low amount of oil for scale commercialization of products of biofuels and having efficient technologies for harvesting microalgae is also recommended. Finally, sustaining algal biofuels is very beneficial for production of biofuels from these factors: land use, balance of carbon, nutrient sources, balance of energy and water.

\section{REFERENCES}

Acien F.G, Fernandez J.M, Molina-Grima E. (2014). Economics of Microalgae Biomass Production. Elsevier B.V. Chapter 14. 314-316. https://doi.org/10.1016/B978-0-444-59558-4.00014-0

Bellou S, Baeshen M, Elazzazy A, Aggeli D, Sayegh F, Aggelis G. (2014) Microalgal lipids biochemistry and biotechnological perspectives. Biotechnol Adv. 32: 1476-93. https://doi.org/10.1016/j.biotechadv.2014.10.003

Bleeke, F., Milas, M., Winckelmann, D., \& Klöck, G. (2015). Optimization of freshwater microalgal biomass harvest using polymeric flocculants. International Aquatic $\quad$ Research, 7(3), 235-244. https://doi.org/10.1007/s40071-015-0108-8

Brennan, L., Owende, P. (2010). Biofuels from microalgae - A review of technologies for production, processing, and extractions of biofuels and coproducts. Renew. Sustain. Energy Rev. 14, 557-577. https://doi.org/10.1016/j.rser.2009.10.009

Bux, F. (2013). Biotechnological applications of microalgae: biodiesel and valueadded products. Boca Raton, FL: CRC Press, Taylor \& Francis Group. https://doi.org/10.1111/jpy.12233

Carpenter, S.R. (2005). Eutrophication of aquatic ecosystems: Bistability and soil phosphorus. Proceedings of the National Academy of Sciences of the United $\begin{array}{llll}\text { States } & \text { of } & \text { 102(29): } & \text { America }\end{array}$ https://doi.org/10.1073/pnas.0503959102

Chen, C.Y., Yeh, K.L., Aisyah, R., Lee, D.J., Chang, J.S., (2011). Cultivation, photobioreactor design and harvesting of microalgae for biodiesel production: A critical review. Bioresour. Technol. 102, 71-81. https://doi.org/10.1016/j.biortech.2010.06.159

Estime, B., Ren, D., \& Sureshkumar, R. (2017). Cultivation and energy efficient harvesting of microalgae using thermoreversible sol-gel transition. Scientific Reports, 7, 40725. http://doi.org/10.1038/srep40725

Davis, R., A. Aden, \& P.T. Pienkos. (2011). Techno-economic analysis of autotrophic microalgae for fuel production. Applied Energy 88(10): 3524-3531 https://doi.org/10.1016/j.apenergy.2011.04.018

Deng, X., Gu, B., Li, Y., Hu, X., Guo, J., \& Fei, X. (2012). The Roles of acylCoA: Diacylglycerol Acyltransferase 2 Genes in the Biosynthesis of Triacylglycerols by the Green Algae Chlamydomonas reinhardtii. Molecular Plant, 5(4), 945-947. https://doi.org/10.1093/mp/sss040

Deng, X., Cai, J., \& Fei, X. (2013). Effect of the expression and knockdown of citrate synthase gene on carbon flux during triacylglycerol biosynthesis by green algae Chlamydomonas reinhardtii. BMC Biochemistry, 14, 38 . http://doi.org/10.1186/1471-2091-14-38

Deng, X., Cai, J., Li, Y., \& Fei, X. (2014). Expression and knockdown of the PEPC1 gene affect carbon flux in the biosynthesis of triacylglycerols by the green alga Chlamydomonas reinhardtii. Biotechnology Letters, 36(11), 2199 2208. https://doi.org/10.1007/s10529-014-1593-3

Ji, C., Mao, X., Hao, J., Wang, X., Xue, J., Cui, H., \& Li, R. (2018). Analysis of bZIP Transcription Factor Family and Their Expressions under Salt Stress in Chlamydomonas reinhardtii. International Journal of Molecular Sciences, 19(9), 2800. http://doi.org/10.3390/ijms19092800

Jorquera, O., Kiperstok, A., Sales, E.A., Embiruc u, M., Ghirardi, M.L., (2010). Comparative energy life-cycle analyses of microalgal biomass production in open ponds and photobioreactors. Bioresour. Technol. 101, 1406-1413. https://doi.org/10.1016/j.biortech.2009.09.038

Kwak, M., Park, W., Shin, S., Koh, H., Lee, B., Jeong, B., \& Chang, Y. K. (2017). Improvement of biomass and lipid yield under stress conditions by using diploid strains of Chlamydomonas reinhardtii. Algal Research, 26, 180-189. https://doi.org/10.1016/j.algal.2017.07.027

Lam, M. K., Lee, K.T. (2012). Microalgae biofuels: A critical review of issues, problems and the way forward. Biotechnol. Adv. 30, 673-690. https://doi.org/10.1016/j.biotechadv.2011.11.008

Lam M. K, Lee K. T. (2014). Scale-Up and Commercialization of Alga Cultivation and Biofuel Production. Chap 12. Elsevier B.V. 256-266 https://doi.org/10.1016/B978-0-444-64192-2.00019-6

Lenka, S. K., Carbonaro, N., Park, R., Miller, S. M., Thorpe, I., \& Li, Y. (2016). Current advances in molecular, biochemical, and computational modeling analysis of microalgal triacylglycerol biosynthesis. Biotechnology Advances, 34(5), 1046-1063. https://doi.org/10.1016/j.biotechadv.2016.06.004

Loera-Quezada, M. M., Leyva-González, M. A., Velázquez-Juárez, G., SanchezCalderón, L., Do Nascimento, M., López-Arredondo, D., \& Herrera-Estrella, L. (2016). A novel genetic engineering platform for the effective management of biological contaminants for the production of microalgae. Plant Biotechnology Journal, 14(10), 2066-2076. http://doi.org/10.1111/pbi.12564

Mata, T.M., Martins, A.A., Caetano, N.S. (2010). Microalgae for biodiesel production and other applications: A review. Renew. Sustain. Energy Rev. 14, 217-232. https://doi.org/10.1016/j.rser.2009.07.020

Pandey, A., Lee, D.J., Chisti, Y., Soccol, C.R. (2014). Biofuels from algae (1st ed.). Amsterdam: Elsevier. https://doi.org/10.1016/C2012-0-00170-6

Park, J.B.K., R.J. Craggs, and A.N. Shilton. (2011). Wastewater treatment high rate algal ponds for biofuel production. Bioresource Technology 102(1): 35-42. https://doi.org/10.1016/i.biortech.2010.06.158

Rabalais, N.N., Turner R.E., Díaz R.J., and Justi D. (2009). Global change and eutrophication of coastal waters. ICES Journal of Marine Science 66(7):1528 1537. https://doi.org/10.1093/icesjms/fsp047

Radakovits, R., Jinkerson, R. E., Darzins, A., \& Posewitz, M. C. (2010). Genetic Engineering of Algae for Enhanced Biofuel Production. Eukaryotic Cell, 9(4), 486-501. https://doi.org/10.1128/ec.00364-09

Razon, L.F., Tan, R.R., (2011). Net energy analysis of the production of biodiesel and biogas from the microalgae: Haematococcus pluvialis and Nannochloropsis. Appl. Energy 88, 3507-3514. https://doi.org/10.1016/j.apenergy.2010.12.052 Salim, S., Bosma, R., Vermuë, M. H., \& Wijffels, R. H. (2010). Harvesting of microalgae by bio-flocculation. Journal of Applied Phycology, 23(5), 849-855. https://doi.org/10.1007/s10811-010-9591-x

Smith, V.H., and D.W. Schindler. (2009). Eutrophication science: Where do we go from here? Trends in Ecology and Evolution 24(4): 201-207. https://doi.org/10.1016/j.tree.2008.11.009

Stephenson, A.L., Kazamia, E., Dennis, J.S., Howe, C.J., Scott, S.A., Smith, A.G., (2010). Life-cycle assessment of potential algal biodiesel production in the 
united kingdom: A comparison of raceways and air-lift tubular bioreactors Energy Fuels 24, 4062-4077. https://doi.org/10.1021/ef1003123

Sturm, B.S.M., and S.L. Lamer. (2011). An energy evaluation of coupling nutrient removal from wastewater with algal biomass production. Applied Energy 88(10): 3499-3506. https://doi.org/10.1016/j.apenergy.2010.12.056

Suali, E., Sarbatly, R., (2012). Conversion of microalgae to biofuel. Renew. Sustain. Energy Rev. 16, 4316-4342. https://doi.org/10.1016/j.rser.2012.03.047

Xu, L., Weathers, P.J., Xiong, X.R., Liu, C.Z., (2009). Microalgal bioreactors: Challenges and opportunities. Eng. Life Sci. 9, 178-189. https://doi.org/10.1002/elsc.200800111

Yang, L., Chen, J., Qin, S., Zeng, M., Jiang, Y., Hu, L., Wang, J. (2018a) Growth and lipid accumulation by different nutrients in the microalga Chlamydomonas reinhardtii. Biotechnology for Biofuels, 11, 40 http://doi.org/10.1186/s13068-018-1041-z

Yang, A., Suh, W. I., Kang, N. K., Lee, B., \& Chang, Y. K. (2018b)

MAPK/ERK and JNK pathways regulate lipid synthesis and cell growth of Chlamydomonas reinhardtii under osmotic stress, respectively. Scientific Reports, 8, 13857. http://doi.org/10.1038/s41598-018-32216-5 